\title{
Interactive comment on "Dissolved organic matter release by phytoplankton in the context of the Dynamic Energy Budget theory" by Eleni Livanou et al.
}

\section{Anonymous Referee \#1}

Received and published: 14 November 2017

The authors develop a model of phytoplankton growth with particular emphasis on dissolved organic matter (DOM) release, based loosely on the Dynamic Energy Budget (DEB) theory. DOM release is described according to the overflow and passivediffusion hypotheses and formulated as balancing the stoichiometries of several cellular compartments. The model is partially calibrated with a dataset and several potential implications are discussed.

General evaluation

This ms leaves me feeling somewhat lost. To begin with, the model appears overly complex compared to the data used for calibration. Table 2 shows more than 40 pa- 
rameters, only slightly less than the number of independent data in Fig. 2. While a few parameters were set to literature values, it still remains difficult to believe that all other parameters could be constrained by the data. This leads to the second major problem, the lack of a proper model validation, which I consider fundamental for any modelling paper. The fact that this applies also to previous publications cited as the foundation for the model further adds to the rather flaky impression of the present ms. The third major problem is in the lack of justification (mechanistic or empirical) or wrong justification of several central model equations. Hence, all conclusions and implications appear unjustified. The last major problem I have with this $\mathrm{ms}$ is that I did not find clear statements about the assumptions, goals, and findings of this study, nor does it appear to add anything really new to the topic of DOM production by phytoplankton. In summary, the study design and model formulation appear so fundamentally compromised that I cannot foresee how this ms could be salvaged within a reasonable number of revisions.

\section{Specific points}

\section{Model complexity and parameter estimation}

The authors use one of several datasets published by Flynn et al. (2008) for calibrating most of the model parameters. They do not specify how the calibration was done but only state that they were tuned. They also do not explain why they chose this particular dataset, although many more exist (some of them cited in the ms). The sensitivity analysis is rather cursory and does not consider parameter covariances nor correlations, for which the authors obviously would have had to consider a much larger dataset. However, the sheer number of parameters considered important (Table S1) would overwhelm any parameter-estimation method I know of. Thus, it is not only a problem of the extent of the dataset but equally one of the complexity of the model formulation.

2. Lack of validation

Printer-friendly version

\section{Discussion paper}


I consider a proper model validation essential to any modelling study. Validation can be, and often is, done together with the model calibration. But this is possible only if the number of estimated parameters is relatively small compared to the number of model variables and also relative to the number of datasets used for calibration. An example of this is the Geider et al. (1998) model (Limnol. Oceanogr.), but it does not apply here. The problem is one of model falsifiability. The large number of sensitive model parameters suggests that this model can be tuned to any kind of data. This means that it has no explanatory power unless the calibrated model is compared to other datasets not used for calibration.

3. Lack of or wrong justification of model equations

Eq. (4) is an unusual form of the light dependence and needs justification. Invoking $\mathrm{NADPH}$ as the agent responsible for $P$ limitation is not very logical as NADPH contains only a tiny fraction of cellular $P$ even under severe $P$ limiting conditions. See, e.g., Ågren's (2004) model in Ecology Letters and refs. therein for a discussion of how $P$ limitation works.

Eqs. (5) and (6) are not explained or derived, nor is a ref. given. I have not seen this form of cell-quota dependence before and wonder why this form, with three or four parameters (depending how they are counted) was chosen over Droop's model, which has only two parameters.

Applying Eq. (7) to $\mathrm{C}$ assimilation does not appear justified. CO2 enters the cell passively, often enhanced by some form of $\mathrm{CO} 2$ concentrating mechanism (CCM) in the chloroplast, so the kinetics are very different from Eq. (7).

I find Eq. (9) problematic for several reasons. First off, it treats the CB cycle as a single enzyme reaction, which is not the case. Secondly, and more importantly, the CO2 concentration inside the chloroplast ist usually kept high enough by the CCM so that RuBisCO is saturated. Thus, the rate of $\mathrm{CO} 2$ fixation is mostly controlled by the rate of light harvesting, as represented here by NADPH arrival rate, and the availability of

Printer-friendly version

Discussion paper 
RuBisCO and the other enzymes of the reaction centers, but not by $\mathrm{CO} 2$ concentration or the substrates for NADPH synthesis.

The use of different handling rates for $\mathrm{NO} 3$ and NH4 in Eq. (10) does not make sense to me. If $\mathrm{DIN}$ (NO3 or $\mathrm{NH} 4$ ) is not limiting the rate-limiting step of $\mathrm{N}$ assimilation is protein biosynthesis, as $\mathrm{NO} 3$ reduction and formation of amino acids are very fast processes. But the maximum rate of protein biosynthesis should not depend on whether the $\mathrm{N}$ derives from $\mathrm{NH} 4$ or $\mathrm{NO} 3$.

NO3 excretion (Eq. 15) does not make sense to me. Maybe one can consider some leakage but most models treat uptake as the net rate already accounting for leakage. I know that some phytoplankton can reduce NO3 to counter photo-inhibition and then release NH4 but I have never seen a report of phytoplankton excreting NO3.

The authors never provide any details how they implemented their model in Matlab. Did they program their own ODE solver or use one of the built-in functions?

The model appears to rest on many implicit, i.e., unspecified, assumptions. Some of the most disconcerting ones concern the internal reserves for $\mathrm{NO} 3$ and $\mathrm{NH} 4$. While phytoplankton may generally have the ability to store $\mathrm{N}$ in the form of amino acids or oligo- or polypeptides etc., storage of inorganic $\mathrm{N}$ has been shown only for very few species, and these are very large and very rare.

While none of these problems are directly related to DOM release, it is clear that the model does not rest on firm grounds. Thus, any calculations regarding DOM release are pointless because they lack a sound underpinning.

4. Lack of clarity, goals, etc.

With respect to the patterns of DOM release and its relation to primary production, it is now generally accepted that these result from interactions among all plankton groups, i.e., bacteria, phytoplankton, zooplankton, detritus, etc. Phytoplankton DOM release is only one of these and while it may well be the dominant source of DOC,

Printer-friendly version

Discussion paper 
whether this applies to DON and DOP as well is still an open question. I think one cannot understand the relation between DOM release and primary production without reference to other processes in plankton ecosystems. But these are missing in the discussion.

In their conclusions, the authors write that their model could be coupled with biogeochemical models (on what scale? 1D, local, global?) but they do not provide any arguments for this anywhere else in the ms. I think a coupling of such a complex model, even if it were theoretically sound, to biogeochemical models is not feasible, simply because it is impossible to constrain its many parameters. Other conclusions are mentioned in the discussion but they appear very speculative. For example, the association of the DOM release mechanism (passive or overflow) with refractory and labile DOM is not explained mechanistically. The way these conclusions are presented, they could equally have been derived directly from observations. It remains unclear how the model relates to them. The point of developing a model, at least as I see it, is to provide insight into how certain mechanisms work, e.g., here it could have been to explain why the overflow mechanism produces more refractory DOC, but this has not been done here. While I do recognise that, technically, the specific model setup is new, I do not see any new insight in terms of mechanistic understanding of DOM release from phytoplankton in this ms.

Interactive comment on Biogeosciences Discuss., https://doi.org/10.5194/bg-2017-426, 2017. 